\title{
La spatialisation de la vie et de la mort dans Depuis toujours, j'entendais la mer d'Andrée Christensen
}

Sarah Abd El-Salam, Université d'Ottawa, Canada

Si la vie est un récit, la mort en serait, aussi un. Mais la mort est avant la vie. Il y aurait donc un récit avant le récit ; un récit sous le récit qui s'écrit - qui le récrit, peut-être en s'écrivant. À moins que nous ne vivions simultanément les deux récits, comme un seul : le récit de la vie de notre mort ; et le récit de la mort de notre vie. (Christensen 7)

Cette citation d'Edmond Jabès, reprise en guise d'épigraphe au roman Depuis toujours, j'entendais la mer d'Andrée Christensen, illustre bien la réflexion métaphysique que celui-ci propose. En effet, la thématique de la vie et de la mort ainsi que le rapport que celles-ci entretiennent avec les personnages, sont au cœur du récit. Lorsqu'Andrea reçoit une lettre de Thorvald, son cousin défunt, jointe à un carnet de notes, la narratrice du récit-cadre est tout d'un coup immergée dans un univers qui, bien que fondamentalement funeste, est marqué par une profonde aspiration à la vie. Par ailleurs, c'est à travers la construction d'espaces symboliques qu'est traitée la problématique de la vie et de la mort dans ce roman ou, plus précisément, dans le récit enchâssé sur lequel portera, en large partie, notre analyse. À un espace-clé du récit correspond donc un système et une charge symbolique qui lui est propre. Les deux espaces que nous analyserons, soit l'eau et la morgue, sont d'abord présentés comme s'ils étaient antagonistes, mais c'est à travers une dialectique symbolique menant à leur enchevêtrement que notre personnage Thorvald atteindra la paix intérieure. Notre étude montrera que la philosophie vers laquelle tend l'ensemble du roman, est au mieux appréhendée à la lumière d'une analyse symbolique de l'espace. Nous examinerons la spatialisation de la vie et de la mort dans l'eau, puis dans la morgue, pour ensuite nous pencher sur le processus de réconciliation, menant ultimement à la fusion des deux symboliques.

\section{L'eau : lieu de contiguïté de la vie et la mort}

L'eau est la première instance de spatialisation de la symbolique de la vie et de la mort. Selon Bachelard, «pour l'imagination matérielle [celle qui s'ancre dans une matière 
élémentaire], tout liquide est une eau. C'est un principe fondamental de l'imagination matérielle qui oblige à mettre à la racine de toutes les images substantielles un des éléments primitifs » (L'eau et les rêves, 158). Retenant cette conception bachelardienne, nous inclurons tout liquide significatif pour notre étude, plus particulièrement trois eaux distinctes que nous étudierons en fonction du rapport qu'elles entretiennent avec la vie et la mort: le liquide placentaire ; l'eau d'Ingelise, définie comme le territoire que celle-ci occupe physiquement et spirituellement (à travers les souvenirs de Thorvald); et enfin l'eau de Katla.

\section{Le placenta}

Le placenta contient la charge symbolique du lieu charnière entre l'état de non-existence (parfois comparé à la mort) et la naissance au monde extérieur. Dans son roman, Christensen propose un déplacement, voire un revirement de cette symbolique couramment admise en associant, dans un premier temps, l'existence dans le placenta à la vie et au bonheur. Les premiers épisodes placentaires relatant l'expérience intra-utérine de Thorvald et de sa sœur jumelle Freya révèlent que cet endroit est empreint de douceur et de béatitude, qu'il s'agit d'un véritable «éden liquide» (38). Thorvald nous raconte qu' « [a]u temps du ventre, encore aveugles, [ils] fais[aient] tout d'abord l'expérience du toucher. La tendresse passait déjà de la menotte de [s]a jumelle à la [s]ienne, [1]e cousait à elle. [S]a peau effleurait son corps, éprouvait le contact d'un autre être humain » (37). Néanmoins, l'harmonie dans laquelle baignent les deux jumeaux, est rompue à la page suivante, lorsque l'accouchement débute. Les contractions de Kirstine, la mère, puis la sortie de Freya du ventre mettent en branle la transformation du milieu édenien en un lieu manifestement oppressant pour Thorvald : "Ma bouche s'ouvrit, mon thorax dilaté, un liquide amer envahit ma bouche, mes poumons, mon estomac. J'étais en train de me noyer. Trente secondes, et la vague se retira, le sang oxygéné me permit à nouveau de respirer. Puis, plus rien. Le silence total après la tempête. J'étais seul pour la première fois. J'eus peur. Très peur » (39). En donnant naissance à Freya, qui décède, Kirstine meurt en couches avant de pouvoir mettre Thorvald au monde. D'ailleurs, personne ne connaissait l'existence de celui-ci avant d'entendre des battements de cœur issus du ventre de la mère morte. Thorvald demeure donc prisonnier du placenta métamorphosé en "sombre caverne, devenue chambre de torture » (40) et ce, jusqu'à ce que le médecin l'en sorte par césarienne. Thorvald, seul survivant, naît donc de la mort, d'où le titre du chapitre : «Naissance de la mort » (33), idée qui le suivra tout au long de sa vie et qui sera renforcée quelques années plus tard lorsqu'il apprendra son 
cannibalisme intra-utérin. À la suite du « cataclysme de sa naissance » (40), Thorvald avoue que «[m]algré le passage des années, le corps n’oublie jamais les traumatismes de la naissance. [...] Oui, je le crois, les tourments de la naissance nous poursuivent jusqu'à la mort » (42) ou, plus précisément, jusqu'à ce que Thorvald se réconcilie avec la vie et la mort.

Cet épisode de l'accouchement, de la vie qui le précède et des deux morts qui s'ensuivent, instaure le mariage symbolique entre le liquide amniotique et toute autre forme d'eau. En effet, au moment même de l'accouchement, «[d]ehors, en proie à de terribles convulsions, la tempête continuait de gronder. Le vent de hurler, respiration courroucée d'un léviathan. La mer se gonflait de vagues furieuses, hurlant comme des chiens en détresse » (40). Cette citation renvoie aux propos de Bachelard qui consacre un chapitre à la colère de l'eau qui, lorsqu'elle «gronde et rugit[,][...] reçoit toutes les métaphores de la furie, tous les symboles animaux de la fureur et de la rage. Son écume ressemble “à la salive d'un léviathan”. [...] Les conclusions d'une naissance dramatique s'élèvent ainsi en puissance jusqu'à être les convulsions d'un océan » (L'eau et les rêves, 230-233). De cette manière, si l'eau est déjà présente lors de l'accouchement, nous apprendrons par la suite que Kirstine et Freya seront mises dans un cercueil pour être jetées à la mer, ce qui unit véritablement l'eau à la mère et à la sœur. Comme ces dernières ont évoqué pour Thorvald la vie et la mort en alternance, nous comprendrons qu'à partir de ce moment dans la diégèse, toute eau acquerra la valeur du placenta et mettra en scène, par conséquent, la même oscillation entre la vie et la mort.

\section{L'eau d'Ingelise}

À la mort de Kirstine, Thorvald est adopté par les employeurs de celle-ci : Erland et Ingelise, qui devient sa seconde mère. C'est notamment à travers la musique qu'ils partagent des moments privilégiés. Toutes les nuits, Thorvald rejoint Ingelise dans la cuisine pour l'entendre jouer du violoncelle et se «laiss[e] bercer par cette voix lumineuse [celle de l'instrument], qui [lui] sembl[e] venir du fond de la mer. Dans les sons liquides, [il] entendai[t] [s]a mère et [s]a jumelle Freya, leur langoureux appel » (58). Si l'appel de l'eau est déjà évoqué dans ces scènes nocturnes, il s'avère d'autant plus flagrant lorsque, chaque matin, Ingelise, non-voyante, compte les pas pour se rendre à l'extrémité de l'île, à côté de la mer, où elle se dévêt avant de jouer du violoncelle. Jour après jour, Thorvald la suit, la regarde et l'écoute en cachette, ce qui lui procure un bonheur incommensurable et lui donne la sensation d'être en vie. Une nuit, Thorvald veut rejoindre Ingelise dans la cuisine, mais celle-ci ne s'y trouve pas. Pris de panique, le jeune 
garçon cherche frénétiquement sa mère adoptive jusqu'à ce qu'Erland le force à aller se coucher. La disparition définitive d'Ingelise est alors à présager; elle sera confirmée par Thorvald le lendemain matin lorsque celui-ci se rend à la mer pour voir si elle s'y trouve, en vain. À cet instant, «[1]e paysage qui [lui] était tant familier était devenu hostile et menaçant sans la présence d'Ingelise » (63). Cette courte citation, si elle montre déjà la transformation de la symbolique de l'eau (par métonymie), enclenche aussi et surtout le mouvement de ressac de cette symbolique, perceptible dans l'extrait suivant :

Assoiffé de présence féminine, je me jetai dans les bras de la mer. Réconfortante, elle m'accueillit, [...] calmant mon angoisse, soulageant ma peine de ses bercements réguliers. Je me lovais dans le liquide de mon embryonnaire mémoire [...]. Je nageais dans le sein de ma mère, mes mouvements s'enroulant autour de mon propre corps. Privé encore d'une autre présence chère, je la retrouvais démultipliée dans l'immensité de l'océan. Ingelise prenait sa place, aux côtés de ma mère et de ma jumelle, transformées en paysage marin.

Soudain, je devins le témoin ébloui d'un spectacle que je ne reverrais jamais. En effet, à chaque déploiement de mes bras, l'eau semblait devenir de plus en plus épaisse et visqueuse. Je nageais au milieu d'une tache immense, douce et gélatineuse, l'eau alourdie par une mystérieuse nappe laiteuse, presque opaque. [...] [L]'eau était entièrement blanche de la laitance des mâles. Des millions, des milliards d'œufs. La mer, de nouveau féconde. La plus vaste nourrice de la planète était redevenue amoureuse. (64-65. Nous soulignons.)

$\mathrm{Si}$, de bienfaitrice lorsqu'il y rejoint Ingelise, l'eau, en l'absence de la seconde mère, est devenue menaçante pour le personnage, elle n'en est pas moins réconfortante lorsqu'il décide de s'y baigner. Thorvald y est entouré par ses deux mères et sa jumelle. Dans la première partie de ce passage, l'eau, imprégnée des fantômes du passé, est donc celle des morts. Toutefois, le retour à la ligne et l'adverbe « soudain » marquent une rupture nette, tant sur le plan typographique que symbolique. La fécondation des poissons engendre un retour à l'eau vitale, féconde. Ainsi, la mer d'Ingelise permet de systématiser la symbolique rattachée à l'eau : celle-ci sera associée tantôt à la vie, tantôt à la mort, mais pas de manière simultanée. Une fois mis en place, ce système symbolique de contigüité ne cessera de s'intensifier. À partir de cet épisode, l'oscillation de l'eau entre la vie et la mort deviendra de plus en plus rythmée et ce, à travers l'eau de Katla.

\section{L'eau de Katla}

C'est à Skagen, ville maritime du Danemark, que Katla et Thorvald décident de se marier après s'être fréquentés quelques temps. Leurs bagages à peine déposés à l'hôtel, ils se rendent 
près de la mer pour commencer «l'exploration de [leur] éden nuptial » (142). Ils avancent de quelques pas dans l'eau et, après un long silence, échangent de brefs vœux avant de fermer les yeux : «[Ils] ven[aient], dans un geste d'abandon absolu, de faire confiance à l'amour, de faire serment de [s']aimer dans la démesure. Ouvrant les yeux, [ils] ét[aient] réellement devenus mari et femme, l'océan pour seul témoin » (145). L'eau, lieu du sacrement marital, fait ici office de chapelle. Une fois cette cérémonie terminée, le couple fait l'amour sur la plage, désireux de «célébrer, au son de l'étrange rumeur des eaux, le mystère qui [les] unissait en une trinité absolue » (146). Toutefois, cette union tripartite, en liant les nouveaux époux à l'océan, les engage, par conséquent, à suivre le mouvement symbolique ambivalent de l'eau. En effet, le matin de leur dernier jour à Skagen, Thorvald veut, avant de repartir, revoir la plage sous «l'envoûtement des eaux qui [1]'avaient soudé à la femme de [s]a vie » (147). Une fois arrivé au bord de l'océan, Thorvald constate que «[1]es eaux se soulevaient lourdement, masses hostiles qui [lui] inspirèrent de la crainte » (148). Alors qu'il est pris d'un malaise, il se rappelle certaines légendes circulant dans la ville «au sujet de ceux qui, au cours des siècles, avaient osé s'avancer de quelques mètres dans les eaux de Skagen; ils devinrent les victimes de ces courants contraires, à l'appétit insatiable et destructeur »(148). Il regarde alors l'océan et aperçoit, subrepticement, sa jumelle qui lui fait signe. De cette manière, l'eau, d'abord seul témoin du mariage, associé à la vie, ne tarde pas à se retransformer en eau des morts avec les légendes et la présence de Freya. Ce retour à l'eau de la mort acquiert alors une portée prophétique en présageant le destin funeste du couple.

Selon le système symbolique mis en place par le roman, l'eau redevient cependant symbole de la vie dans un épisode subséquent : celui près de l'étang à Endelave. Lors d'une visite à Erland, Thorvald et Katla se rendent près de l'étang, où, tapis sur le sol, ils assistent à un spectacle naturel inattendu: la danse d'amour des grues cendrées. Dans une volonté de communier avec cette nature, les deux personnages se donnent l'un à l'autre. Quatre semaines plus tard, Thorvald apprend que dans «[h]uit mois, [...] [il] ser[a] père. » (156) Tout comme la mer d'Ingelise, l'eau est ici à nouveau féconde et préside à la conception d'une petite fille portant le même nom que la jumelle de son père : Freya.

Le mouvement de balancier de l'eau entre la vie et la mort atteindra toutefois son paroxysme plus tard dans la narration avec la noyade de Katla et de sa fille Freya. Se sentant menacé par le pouvoir grandissant des Nazis au Danemark, le couple avait déménagé à Endelave 
pour protéger Katla qui était enceinte. Ses traits physiques pouvaient effectivement rappeler ceux d'une Juive ou d'une Tzigane et Thorvald avait jugé qu'ils seraient en sécurité dans son île natale. Quelques années après la naissance de Freya, Katla, pour des raisons qui demeurent obscures (le récit suggère toutefois qu'elle désirait peut-être s'échapper du Danemark occupé), monte à bord d'un bateau et amène sa fille, à l'insu de Thorvald. La tempête gronde et celui-ci part à leur recherche. Il arrive trop tard. La mer, furieuse

aspira le voilier, le tint suspendu dans l'air, puis le lâcha, le projetant dans l'abîme. Une autre vague se gonfla, se gorgea, relança dans l'air la frêle embarcation. Les éclairs et le tonnerre attaquaient sans relâche les eaux noires et frappèrent le mât brisé du Koerlighed. Puis, se produisit l'inimaginable des pires cauchemars. [Thorvald] vi[t] Katla lancer [leur] enfant dans la gueule infernale de la mer, qui n'en fit qu'une bouchée. Une boule de feu illumina le ciel. Le voilier, devenu bateau des morts, coula. [...] Une vague monstrueuse se dressa et [..] rejeta [Thorvald] contre le rocher. [...] [Il] sombr[a] dans le noir. (172)

Plusieurs mois après cette tragédie, Thorvald retourne à la mer : « [Il] aurai[t] dû se sentir apaisé, mais la mer avait cessé d'être à [s] es yeux l'accueillante créature-abri qui avait bercé [s]on enfance. [...] Elle n'était plus que gouffre monstrueux, hypocrite tombeau à ciel ouvert » (176). Thorvald proclame sa rupture avec l'eau lorsqu'il

regard[a] la mer avec haine. [II] la trouv[a] laide, hideuse et méchante, [s]'indign[a] à la voir se gonfler, orgueilleuse et implacable. [II] aurai[t] voulu d'un simple regard l'arrêter, commander son retrait, la punir. [II] méprisai[t] la fureur exécrable, la gueule écumante de cette immense bête de mort qui vit de la misère des humains. [...] [Il] brandi[t] les poings, prêt à frapper l'ennemi. Avec toute la rage qui [lui] dévorait le cœur, l'âme et l'esprit, [il] crach[a] dans les vagues. (176-177)

À travers ce geste violent, Thorvald met fin au système de contigüité de la vie et de la mort dans l'eau. Celle-ci ne représentera dorénavant que la mort et ce, jusqu'à la réconciliation finale. En évacuant la vie pour ne conserver que la mort, la symbolique de l'eau s'en trouvera sclérosée. En effet, puisque l'eau renvoie au placenta, il est essentiel au personnage qu'elle incarne aussi bien la mort que la vie. On comprendra toutes les références maternelles et placentaires lorsque Thorvald se réfugie dans l'eau. Tel que l'énonce Bachelard, «[1]'eau nous porte. L'eau nous berce. L'eau nous endort. L'eau nous rend notre mère » (L'eau et les rêves, 178). En accusant la mer de tous ses maux et en décidant de rompre avec le réconfort maternel qu'elle lui procurait, Thorvald renonce à son éden originel pour ne garder qu'une vision funeste de la vie. Ainsi, afin 
d'atteindre un équilibre intérieur, la philosophie de Thorvald devra connaître une dialectique entre cette symbolique de l'eau et une autre, tout aussi fondamentale : celle de la morgue.

\section{La morgue : lieu de cohabitation de la vie et de la mort}

Si l'eau s'avère l'espace où la vie et la mort se fréquentent de manière contigüe jusqu'à la scène de rupture, la morgue où Erland exerce son métier se présente comme le lieu de conjonction et de cohabitation simultanée de la vie et de la mort, où règne la devise : «Mortui vivos docent (Les morts enseignent aux vivants) »(86). La description de cette morgue nous donne à voir un espace clos presque atemporel : «[f]ormant un cercle, des arbres privés de leurs branches les plus basses, troncs lisses comme le marbre des colonnes d'un temple. Au cœur de ce sanctuaire, aéré et majestueux [...] se dressait la morgue, humble cabane de cèdre, semblable à une demeure primitive»(86). Ce terme de «demeure primitive » nous renvoie à la hutte primitive, qui, selon Bachelard dans sa Poétique de l'espace, « est la solitude centrée. [...] Autour de cette solitude centrée rayonne un univers qui médite et qui prie, un univers hors de l'univers. La hutte ne peut recevoir aucune richesse "de ce monde". [...] De dépouillement en dépouillement, elle nous donne accès à l'absolu refuge » (46). La morgue est un «univers hors de l'univers ", car c'est dans cet endroit, communément admis comme celui de la mort, que Thorvald en apprendra davantage sur la vie à travers deux épisodes marquants : celui de son initiation à la thanatopraxie et celui de l'accouchement de son épouse Katla.

\section{L'initiation}

$\mathrm{Au}$ moment où Thorvald atteint la puberté, Erland décide de lui faire passer un rite initiatique se déroulant dans la morgue. Avant d'en franchir le seuil, le père adoptif prononce un avertissement :

Maintenant, Thorvald, concentre-toi ! La porte que je vais bientôt pousser est l'ouverture fondamentale de l'expérience. Porte d'enfer ou porte d'or, il n'en tient qu'à toi. Une fois de l'autre côté, plus de retour possible. Tu franchis ce passage en enfant, mais tu n'en ressortiras pas de même. Ce que tu y observeras, peu d'humains le voient dans leur vie. Au-delà des terreurs enfantines, tu prendras conscience du drame immense de la vie et de la mort. N'oublie jamais cette phrase : entrer chez les morts, c'est découvrir la vie. Pardelà ce seuil, la vie et la mort ont la même valeur. (86. Nous soulignons.)

À la suite de ces paroles aux sonorités quasi-prophétiques qui seront au demeurant la clé de voûte de cette scène, Erland enseigne à Thorvald ses techniques de thanatopraxie. Le jeune 
homme devra ensuite embaumer le prochain corps sans la présence de son second père. Thorvald se rend rapidement compte qu'il s'agit de celui de Lisbeth, sa grande amie d'enfance qu'il avait perdue de vue quelques années auparavant et dont il ignorait jusqu'alors le décès. Une fois le choc initial passé, Thorvald, suivant les enseignements d'Erland, doit attester médicalement la mort de Lisbeth. Il lui faut donc la déshabiller. N'ayant jamais connu le corps d'une femme, Thorvald sent l'excitation monter en lui, qui se traduit par une gradation dans l'extrait suivant :

J'étais saisi de fébrilité à l'idée de devoir déshabiller Lisbeth pour constater son décès, confus d'avoir à observer son corps nu. [...] Était-ce uniquement par impatience de mettre en pratique les techniques du métier, nouvellement apprises, que je sentais monter en moi une fièvre aussi ardente? [Il commence à la déboutonner] [...] Saisi d'une rage qui me surprit, j'arrachai les derniers boutons de nacre du sous-vêtement qui m'empêchaient de voir la peau de la défunte. Puis, d'un geste presque violent, je déchirai la soie légère qui la couvrait encore. Ébloui par tant de fraîche beauté, [...] [m] û par des impulsions irrépressibles, mes doigts envoûtés s'aventurèrent sur les rondeurs invitantes, ma paume prenant plaisir à les envelopper, mes doigts, à les faire apparaitre, puis disparaître. (103-104. Nous soulignons.)

Cet épisode, dont le rythme scandé, alternant douceur et violence, fait écho au rituel, se poursuit jusqu'à la première jouissance de Thorvald qui, avant son orgasme, avoue que « [1]a vie bouillonnait en [lui] si fort, qu['il] en vin[t] à oublier complètement la mort » (105). Se rendant compte de la portée nécrophile de son acte, le jeune homme nous explique qu' « [a]veuglé par l'envoûtement de [s]a première découverte du corps de la femme, [il] [s]'étai[t] laissé duper par cette dernière vague de vie sentie dans un être qui avait cessé de respirer, mais qui n'était pas tout à fait prêt à être livré à la mort. [II] avai[t] cherché à posséder l'étincelle qui refusait de s'éteindre » (109). Thorvald termine alors la toilette mortuaire de Lisbeth. Lorsqu'Erland revient à la morgue, l'embaumement ainsi que le rite initiatique sont achevés. Thorvald, maintenant adulte, et son père quittent la «hutte primitive, antre du monstre, nuit cosmique, où [le jeune homme] venai[t] de vivre une mort et une naissance rituelles. Le sentier du retour avait changé, la nature avait perdu sa légèreté » (111). À travers cet épisode, le protagoniste fait, pour la première fois, l'expérience d'une pulsion sexuelle intrinsèquement vitale dans un endroit habituellement associé à la mort. La morgue, comme l'avait prédit Erland, devient l'espace de cohabitation de la vie et de la mort, l'une y étant indissociable de l'autre. Si la morgue est le lieu de la première jouissance, il ne sera donc pas étonnant qu'elle soit aussi celui de la naissance de Freya. C'est effectivement dans ce lieu d'alliance indissoluble entre la vie et la mort 
qu'accouchera Katla.

\section{L'accouchement}

Tel que nous l'avons déjà souligné, la grossesse de Katla coïncide avec le début de la déportation des Juifs au Danemark qui pousse les mariés à emménager à Endelave, village plus en retrait qui assurera leur sécurité. Lorsque Katla commence à ressentir des contractions, Thorvald, de peur que des espions se soient dissimulés dans les alentours, juge plus prudent de ne pas appeler le médecin ou la sage-femme. Comme Erland a une formation en médecine, Thorvald décide d'emmener Katla à la morgue afin qu'elle y accouche. Il court donc «à l'étable prendre les chevaux et le corbillard » (162). Évoquant presque une scène christique renversée, c'est dans cet attelage qu'ils arrivent à la morgue, où, rappelant la trinité, trois tables sont disposées. Deux d'entre elles sont occupées par des cadavres attendant d'être embaumés. On installe Katla sur la troisième. À ses côtés, une main cadavérique dépasse du drap et le rapprochement entre la vie et la mort s'opère déjà à travers un parallèle syntaxique : « À côté de la main du cadavre [d'une femme], celle fiévreuse, aux doigts roses, de Katla » (164). Au comble de la douleur causée par l'accouchement, au moment même où le bébé est sur le point de naître, «Katla saisit le premier objet à sa portée. La main du cadavre. Elle poussa un dernier hurlement animal, puis relâcha la main inanimée » (164). Bien que le symbole de la vie tenant la main de la mort puisse paraître un peu banal, il n'en demeure pas moins que la main de ce cadavre féminin aide et accompagne Katla dans sa dernière poussée. Ainsi, deux femmes, l'une morte, l'autre au paroxysme de la vie, donneront naissance à une petite fille, d'où le titre du chapitre : « La mort, sage-femme de la vie » (157). Erland, ne disposant pas de langes à proprement parler, habille Freya d'un linceul et le couple reprend le corbillard pour rentrer à la maison. Le vêtement improvisé de l'enfant condense la symbolique associée à la morgue : la vie enveloppée dans la mort.

Dans ces deux épisodes, nous constatons que la morgue, lieu de la mort par excellence, s'avère, en même temps, le lieu des pulsions vitales. Associant d'abord la mort à la vie (la première jouissance relevant de la nécrophilie) et ensuite la vie à la mort (accouchement aidé par la morte), la morgue devient le lieu où la vie et la mort s'équivalent et deviennent interchangeables. De plus, cet espace, bien qu'il mette en scène l'accouchement, demeure un endroit essentiellement masculin. Bien entendu, nous penserons à l'orgasme de Thorvald qui s'y déroule et qui est le seul dans le roman à nous être décrit aussi explicitement. De manière plus 
spirituelle, il s'agit du lieu de prédilection d'Erland où celui-ci pratique ses deux arts : la thanatopraxie et le dessin, qui s'avèrent représentatifs de la philosophie du père. En effet, celui-ci ne comprend pas que les morts inspirent la peur chez autant de gens. Selon lui, on oublie trop souvent que les morts ont été des êtres vivants et aimés, car dès que «la vie les quitte, on les renie, comme s'ils étaient des êtres différents, de dangereux étrangers. La raison cherche à écarter les morts, à les refouler de la vie consciente, avec les morts, on enterre les pensées de la mort, le cadavre n'étant guère plus qu'un douloureux rappel de notre trop bref passage sur terre » (90-91). Après avoir embaumé les cadavres, Erland dessine leur visage avant que les dernières étincelles de vie ne quittent leurs yeux. Un jour, celui-ci se confie à Thorvald, qui s'interroge sur l'art de son père :

C'est ma manière [...] de les honorer. Je me suis donné pour mission de dévoiler leur beauté qu'on refuse de voir. Je lutte contre l'indifférence. Cela me blesse lorsqu'on inflige aux défunts une seconde mort en les laissant sombrer dans la fosse commune de l'oubli. Le comble de l'injure est de ne même pas se souvenir qu'on les a oubliés. Je suis leur mémoire vivante, et mes dessins sont les monuments qui en témoignent. [...] On ne peut pas simplement laisser l'âme des morts s'évaporer dans l'atmosphère, frissonnant dans la nudité de son origine. Mon rôle est de la saisir avant qu'elle ne quitte définitivement le corps, d'en capter sur papier les derniers éclats de lumière, pour lui en faire un linceul d'éternité. Mes yeux, mes mains, artisans à son service. (89-90)

La morgue se veut donc la représentation symbolique d'un idéal et de la philosophie du père.

Alors que la symbolique de la morgue soude la vie à la mort dans un espace clos et masculin, celle de l'eau, nous l'avons vu, est un lieu davantage maternel et féminin, ouvert et marqué par l'alternance de la vie et de la mort. On comprendra donc pourquoi Erland « avait fait construire sa morgue, la dissimulant derrière de grands arbres, loin du passage d'Ingelise, qui avait préféré vivre dans l'ignorance du métier de son époux » (85) et qui n'acceptait pas la présence des dessins mortuaires dans la maison familiale. Ainsi, «[t]iraillé par ces points de vue opposés de [s]es deux parents adoptifs, [Thorvald] faisai[t] [s]on apprentissage de la mort dans une inconfortable confusion qui, peu à peu, façonnait [s] a conception même de la vie » (49). Afin de permettre au protagoniste de trouver sa paix intérieure, la narration devra donc, de manière symbolique, concilier ces deux manières de spatialiser la dynamique entre la vie et la mort.

\section{La réconciliation}

La quête métaphysique du personnage ne peut s'effectuer que si vie et mort 
s'enchevêtrent, comme dans la morgue, mais dans le sein maternel de l'eau. Pour ce faire, Thorvald devra d'abord faire son deuil en amnistiant l'eau et en l'acceptant à nouveau comme source de vie. Cette réconciliation se déroulera en trois étapes.

La neige : l'eau-cimetière

Quelques mois après le décès de sa femme et de sa fille pendant lesquels il était resté enfermé dans la maison familiale, Thorvald décide de se rendre au cimetière d'Endelave alors qu'il neige. Bien que Katla et Freya n'y aient pas leurs sépultures, la mer n'ayant jamais rendu leurs corps, Thorvald s'assoit devant un amas de tombes. Peu à peu, la neige le recouvre, le transformant à son tour en tombeau :

Mes vêtements devinrent vite blancs, linceul de paix. J'écoutais la neige ensevelir mon corps, me remplir l'âme. J'étais une pierre tombale de chair et de sang, dressé au-dessus des morts. Mon esprit vide s'oubliait, se laissait remplir de leur calme présence. Mon corps disparu, je rêvais avec mes morts. Ensemble, vivants et défunts dans le partage d'un même néant, solennellement consacré par le silence. Autour de moi, le temps, mort. Je sombrai dans le sommeil. Une eau fraîche qui ruisselait le long de mes joues me réveilla. Un chaud rayon de soleil avait fait fondre la glace agglomérée sur mes sourcils. [...] Recouvert de neige, je ressemblais aux monuments funéraires qui m'entouraient. (185)

Il est intéressant de constater que cette scène de recueillement se déroule dans la neige et non dans l'eau à proprement parler, malgré qu'elle en soit un avatar. Cet extrait renvoie implicitement à Katla puisque Thorvald l'a nommée ainsi en fonction du volcan éponyme. Celuici, composé de lave et d'une calotte glaciaire au contact desquelles se forme un lac, comprend les trois états de l'eau lorsqu'il entre en éruption. De la même manière, la glace accumulée sur les sourcils de Thorvald fond au contact du soleil et ce sont les gouttes d'eau qui sortent ce dernier de sa torpeur. Une fois réveillé et avant de se lever, Thorvald

traç[a] sur l'amas de neige recouvrant [s]es cuisses les mots In memoriam. [II] [s]e dress[a] péniblement, ankylosé par les heures d'inertie. Monument vivant, [il] circulai[t] entre les tombes inertes et muettes. La voix de [s]es morts résonnait en [lui], cristalline, leur respiration bien vivante respirait dans la [s]ienne. «Mes absents sont enfin en moi, je n'ai plus à les chercher. C'est moi leur cénotaphe, et je suis devenu leur mémoire, [s'est-il] dit. » Dans un état de grâce, [il] quitt[a] le cimetière, prêt à retourner chez les vivants. (186)

Le personnage pense ici avoir fait son deuil en laissant ses morts fusionner avec lui. Contrairement à ce que peut croire Thorvald, cet épisode est fondamental dans son processus de 
guérison parce qu'il en marque la première étape, mais non pas la finalité. En effet, tel que nous l'avons vu, le deuil réel du protagoniste ne peut passer que par l'acceptation de l'eau. Etant donné que la neige est un état de l'eau ici médiatisée, cette première démarche de réconciliation ne s'avère que partielle. Thorvald, se croyant cependant rétabli, quitte Endelave, où il ne retournera que quelques années plus tard.

\section{La méduse ou le retour à l'eau vitale}

Lors de son retour à son île natale, sept ans après l'épisode du cimetière, Thorvald ne se rend pas à la mer, ce qui nous laisse déjà présager l'incomplétude de son deuil. Cette impression sera confirmée lorsqu'Erland emmène son fils adoptif sur l'étang afin qu'ils partagent un repas. L'eau est restée, pour Thorvald, celle des morts :

L'étang devenait l'eau-mère du chagrin, matière même de la mélancolie, qui me renvoyait, dans de lugubres murmures, de sombres épisodes de ma vie passée. Notre légère embarcation se remplissait des spectres de mon existence, de mes regrets et de mes remords, véritable barque de Charon qui menaçait de sombrer et de m'ensevelir dans son enfer fluide. Un épisode douloureux de mon enfance me remonta à la gorge. Encore une autre mort se réveillait dans les eaux troubles du souvenir. (211)

Le champ sémantique de la mort l'emporte et si l'eau est qualifiée de « mère ", il ne s'agit que de celle du chagrin et de la mélancolie. Ce n'est que quelques jours plus tard que Thorvald reçoit l'appel de la mer après avoir aidé Erland à préparer un bébé défunt pour sa séance de photographie. À l'instar de l'initiation dans la morgue, l'avancée de Thorvald vers la plage nous est relatée de manière très rythmée et déploie de nombreuses stratégies qui établissent une tension narrative. La répétition-variation du titre, « [a]u loin, j'entendais mugir la mer », suivie du questionnement de Thorvald : «Ou était-ce mon sang qui grondait ? (225) entraîne le début du fusionnement de Thorvald et de l'eau. Le personnage revit physiquement sa propre naissance lorsqu' " un liquide amer envahit [s]a bouche, [s]es poumons, [s]on estomac. [Il] étai[t] en train de [s]e noyer. De grosses mains moites [1]e frappaient dans le dos. Une fois, deux fois, trois fois, elles répétèrent le même manège. Étai[t-il] en train de naître, ou au seuil de la mort ? » (225). La marche de Thorvald est scandée par le nombre de pas qu'il effectue et atteint son paroxysme quand celui-ci «pouss[a] le cri qu['il] n'avai[t] pas poussé à [s]a naissance. [Il] hurl[a] jusqu'au bout de [s]on souffle. Jusqu'au bout de [s]on âme. Épuisé, [il] gagn[a] enfin la plage » (225). Cette expérience physique, voire mystique, le prépare à se réconcilier avec l'eau maternelle. Arrivé sur la plage, il voit une méduse échouée, à laquelle il se compare. L'utilisation du 
symbole de la méduse n'est pas anodine. Étant la seule des trois gorgones à être mortelle, Méduse symbolise «l'ennemi à combattre [...], la pulsion spirituelle et évolutive [...] l'image déformée de soi »(Chevalier et Gheerbrant 482). Elle pétrifie ceux qui la regardent, car elle leur renvoie le reflet de leur propre existence. En décidant de ramener la méduse à la mer, Thorvald se sauve donc lui-même, avant tout. Il s'approche des flots et dépose la méduse sur son torse. Celle-ci, qui reprend peu à peu vie, laisse une brûlure sur le corps du personnage, mais «la douleur vive s'estompa rapidement sous l'effet calmant de la vague, fraîche et caressante » (229). En tendant à Thorvald leurs «bras réconciliateurs» (nous soulignons), les « eaux maternelles » (230) regagnent leur charge symbolique de la vie. Pendant qu'il se laisse bercer par la mer retrouvée, le personnage ressent sur son torse «la trace rouge vif des cheveux de la méduse, signe qu'il fallait toujours donner une autre chance à la vie, au risque de se blesser. Oui, [...] la vie est une douce brûlure » (230). Cet épisode marque donc la réconciliation de Thorvald avec l'eau.

Sur le plan de la symbolique de l'eau, la diégèse est construite en crescendo qui culmine en cette scène (ce qui est renforcé par le décompte des pas et les effets physiques de la naissance, notamment l'étouffement dû au placenta). Cet épisode permet au personnage d'accepter pleinement sa naissance dans la mort, car en poussant le cri qu'il n'avait pas émis à sa naissance, le personnage naît, cette fois-ci véritablement. À partir de cet instant, Thorvald peut donc se diriger vers sa mort, ou plutôt vers la naissance de sa mort. À présent, la symbolique de l'eau est prête à accueillir celle de la morgue pour former une symbiose entre la vie et la mort, nécessaire à la quête spirituelle du personnage.

\section{L'eau-morgue}

Quelques années plus tard, Erland décède des suites de la maladie d'Alzheimer, à travers laquelle Thorvald n'a cessé de l'accompagner. Notons que le père ne meurt pas dans l'eau, mais dans sa chambre, ce qui s'avère crucial sur le plan symbolique. En effet, si le décès d'Erland avait eu lieu dans la mer, l'on peut se douter que l'eau serait redevenue amère pour Thorvald qui devrait à nouveau se réconcilier avec elle. Après avoir embaumé son père adoptif, dernier rituel du roman, Thorvald, à la demande d'Erland, réunit les dessins mortuaires. Il se rend au bord de l'eau et «un à un, couch[a] dans cette morgue à ciel ouvert [...] le visage de centaines d'hommes, de femmes et d'enfants d'Endelave »(260. Nous soulignons). La dispersion dans l'eau des dessins, rattachés à la symbolique de la morgue, entraîne, à cet instant précis dans le 
récit et sans précédent, la périphrase «morgue à ciel ouvert » pour désigner la mer. La fusion de la morgue et de l'eau est renforcée par le sentiment de Thorvald lorsqu'il regarde les dessins sur les flots : «Cette cérémonie des adieux me fendit le cœur ; à cet instant, je sentis Erland quitter définitivement notre monde » (261). Erland et ses dessins, renvoyant à la morgue, sont à présent dissous dans l'eau et c'est avec cet épisode que commence véritablement le processus de symbiose entre les deux pôles symboliques.

Peu à peu, Thorvald se rend compte de sa vieillesse. Dorénavant, plus rien ne le retient sur terre et, fatigué de ses nombreuses renaissances, il apprend à «enfanter [s]a vieillesse, à tutoyer avec patience la déchéance de [s]a mort qui approchait à pas sûrs, à pardonner à [s]on corps de [lui] ravir peu à peu la vie »(263). Acceptant la vieillesse et sa mort imminente, Thorvald se met à apprécier les choses simples de la vie. Il adopte la philosophie d'Erland (celle de la morgue), dans laquelle vie et mort sont inséparables; il «célébrai[t] la chance d'être vivant, de témoigner chaque matin de la naissance de la lumière. Du même coup, [il] recevai[t] la vie elle-même, non comme le rythme tonifiant d'une mer impétueuse, mais comme l'apaisant flux et reflux de la vague »(265). En accueillant la vie, Thorvald est à présent disposé à préparer sa propre mort, qu'il désire devancer. Affranchi de l'oppression de sa naissance dans la mort, il organise avec soin la naissance de sa mort : «Ce désir de mourir, je l'ai nourri pendant neuf mois, comme une mère nourrit l'enfant qu'elle porte en elle. Il est maintenant temps de mettre au monde ma mort. D'être son géniteur, son poète, son artiste » (268). Un jour, Thorvald se laisse donc emporter par l'eau. Cette intrusion du personnage masculin dans l'élément féminin complète la fusion de la morgue à l'eau et marque l'aboutissement de la quête spirituelle de Thorvald. Celui-ci a accepté la mort dans sa vie et la vie au seuil de sa mort. Son suicide couronne l'alliance indissoluble entre la vie et la mort, qui permet une paix intérieure.

En revanche, cet achèvement spirituel n'est pas une fin en soi. La fusion de la vie et de la mort donne lieu à une réelle transcendance, voire à une apothéose. Le dernier chapitre du récit enchâssé qui narre le suicide de Thorvald, s'avère le treizième. Or, le roman consacre un chapitre entier à l'arcane du Pendu dans le jeu du Tarot. La cartomancienne qui y est mise en scène, indique à Thorvald que la douzième carte, celle du Pendu, est tout de suite suivie par celle de la Mort. Elle insiste cependant sur la signification de ce dernier arcane qui ne renvoie pas à la mort en tant que telle, mais plutôt à la renaissance. Ce dernier et treizième chapitre, s'il se clôt sur le suicide du personnage principal, relate, en fait, l'ultime renaissance, l'«Ultima verba » 
(275). Cette transcendance de Thorvald est mise en relief par l'emploi du futur simple dans ce chapitre. Elle est également amplifiée par le retour au récit-cadre à la fin du roman. Andrea, ayant terminé l'écriture du livre que nous lisons, Depuis toujours, j'entendais la mer, retournera à Endelave et en déposera un exemplaire sur les flots. Le roman-tombeau, bien qu'il renvoie au genre même de l'œuvre, devient un Totenbaum, tombeau matériel de Thorvald. Les effets d'enchevêtrement se démultiplient : la morgue dans l'eau, la vie dans la mort (et inversement), le récit dans le récit, le roman, œuvre d'art, au sein d'un livre, tombeau physique. Ces emboîtements permettent l'accès à l'infini, mot sur lequel se conclut le roman.

La réflexion, voire la philosophie, que nous livre Christensen, prend son essor dans la spatialisation de la vie et de la mort qui permet d'opposer, pour mieux rallier, deux différentes manières d'appréhender ces préoccupations ontologiques. L'apothéose de Thorvald, résultant de la dialectique de celles-ci, entraîne la confusion des voix narratives à la fin du roman. Andrea, dans sa lettre à Thorvald, lui confie : «J'ai été étonnée d'être bouleversée par vos paroles, et encore aujourd'hui, d'être émue en lisant vos mots dans les miens, confondus au point où je ne sais plus qui a écrit quoi, tant nos voix sont à l'unisson. [...] Oui, deux labyrinthes se sont croisés et se sont imbriqués l'un dans l'autre » (284-285). L'épilogue, en plus de souder Andrea et Thorvald, brouille la frontière entre la narratrice, figure auctoriale du récit-cadre, et l'écrivaine elle-même. Si des propos tels que «l'écriture de ce livre fut une longue veillée funèbre » (284) et «Connaissez-vous le prix de la gestation et d'un accouchement qui durent six ans ?» (287) sont signés de la main d'Andrea, ils sont aussi attribuables à Andrée Christensen. Cette fusion des voix donne lieu à un discours métatextuel portant sur le processus de création qui permet, non pas d'échapper à la mort, mais de transcender son emprise angoissante. En ce sens, la conception de l'art se veut symptomatique de la réflexion proposée dans le récit, telle qu'illustrée par la citation de Georges Didi-Huberman reprise par Andrea (ou Andrée) : «Les choses de l'art commencent souvent à rebours des choses de la vie. La vie commence par une naissance, une ouvre peut commencer sous l'empire de la destruction : règne des cendres, recours au deuil, retour des fantômes, nécessaire pari sur l'absence » (286-287). Ainsi, bien que «[l]e paradoxe vie-mort porte en lui un mystère qui n'est peut-être pas fait pour être élucidé » (285), l'écriture touchante et poétique de Christensen accompagne le lecteur dans une exploration de soi, d'abord ébranlante, mais menant peu à peu à la plénitude. Après tout, «[l]'agonie de tous ne repose-t-elle pas en chacun de nous? » (289) 


\section{Bibliographie}

Bachelard, Gaston. L'eau et les rêves. Paris : José Corti, 1976 [1942].

---. Poétique de l'espace. Paris : PUF, 1964 [1957].

Chevalier, Jean et Alain Gheerbrant. Dictionnaire des symboles. Paris : Laffont/Jupiter, 2005. Christensen, Andrée. Depuis toujours, j’entendais la mer. Ottawa : David, 2007.

Lacombe, Gilles. « La mer transfigurante ». Liaison 136 (2007) : 64. 